\title{
Numerical Investigation of Injection, Mixing and Combustion in Rocket Engines Under High-Pressure Conditions
}

\author{
Christoph Traxinger, Julian Zips, Christian Stemmer, and Michael Pfitzner
}

\begin{abstract}
The design and development of future rocket engines severely relies on accurate, efficient and robust numerical tools. Large-Eddy Simulation in combination with high-fidelity thermodynamics and combustion models is a promising candidate for the accurate prediction of the flow field and the investigation and understanding of the on-going processes during mixing and combustion. In the present work, a numerical framework is presented capable of predicting real-gas behavior and nonadiabatic combustion under conditions typically encountered in liquid rocket engines. Results of Large-Eddy Simulations are compared to experimental investigations. Overall, a good agreement is found making the introduced numerical tool suitable for the high-fidelity investigation of high-pressure mixing and combustion.
\end{abstract}

\section{Introduction}

In the design and development process of new generation rocket engines computational fluid dynamics (CFD) has become an indispensable tool. By means of CFD, the turbulent injection, mixing and combustion process inside the combustion chamber can be studied in detail providing information about, e.g., the mixture preparation and the combustion efficiency. The extreme operating conditions found in liquid rocket engines (LREs), in particular the high pressures of more than 100 bar and the large temperature range covering three orders of magnitude, are very challenging and demand for high-fidelity and at the same time robust approaches for both the flow solver and the closure models. In terms of thermodynamics, the fluid shows signifi-

\footnotetext{
C. Traxinger $(\varangle) \cdot$ J. Zips $\cdot$ M. Pfitzner

Institute for Thermodynamics, Bundeswehr University Munich,

Werner-Heisenberg-Weg 39, 85577 Neubiberg, Germany

e-mail: christoph.traxinger@unibw.de

\section{Stemmer}

Chair of Aerodynamics and Fluid Mechanics, Technical University of Munich,

Boltzmannstr. 15, 85748 Neubiberg, Germany

e-mail: christian.stemmer@tum.de
} 
cant real-gas effects and therefore the state variables depend on both the temperature as well as the pressure. The nonideal fluid behavior results in strong nonlinearities which have to be properly handled by the flow solver. For an appropriate representation of the combustion process, turbulence-chemistry interaction has to be taken into account as the high-pressure conditions imply very thin reaction zones which cannot be resolved in the context of Large-Eddy Simulations (LESs).

Over the past two decades, different research groups have put a lot of effort into the development of CFD frameworks [16]. Thereby, important milestones have been achieved using Direct Numerical and Large-Eddy Simulations: Starting in 1998, Oefelein and Yang [17] were the first to conduct LESs of reacting flows of gaseous hydrogen/liquid-like oxygen under supercritical pressure conditions and showed the effect of the pressure on near-critical mixing and combustion. Zong et al. [37] investigated the injection of liquid-like nitrogen into itself and found that the large density stratification results in enhanced axial and dampened radial flow oscillations. In 2006, Bellan [3] studied a binary heptane-nitrogen mixing layer and pointed out the importance of transport and turbulence modeling for an appropriate representation of the mixing process. In the subsequent years, different groups followed the example of these groundbreaking investigations and conducted detailed studies of the combustion process under LRE conditions: Amongst others, Ribert et al. [23] focused on the dependency of the flame thickness and the heat release on pressure and strain rate and quantified the influence of Soret and Dufour effects. Lacaze and Oefelein [12] performed a detailed analysis of strain effects, pressure and temperature boundary conditions as well as nonideal fluid behavior on the flame structure in both physical and mixture fraction space to develop a tabulated combustion model.

Although a huge research effort was already undertaken to understand the process of injection, mixing and combustion in LREs, many open questions are still remaining. In the context of thermodynamics, recent experimental investigations question the assumption of a solely single-phase state under LRE conditions. For instance, Roy et al. [24] injected initially supercritical fluoroketone into a supercritical nitrogen atmosphere and reported the presence of droplets and ligaments at the periphery of the jet at sufficiently low ambient temperatures indicating mixtureinduced phase separation. Similar findings were also reported by, e.g., Muthukumaran and Vaidyanathan [15]. With regard to high-pressure combustion, the shift towards methane-fired, reusable rocket engines was a game changer not only from an economical point of view but also for the development of reliable CFD tools. As a result of the large number of species involved in methane combustion, tabulation methods are useful allowing an efficient and reliable numerical investigation of LREs. Due to the increased importance of the mechanical integrity in reusable LREs, the understanding and prediction of flame-wall interaction has become a crucial point of future high-pressure combustion investigations. In addition, the understanding of flame-flame interaction is also of high relevance for the design process of future LREs as methane-fired engines have not been flown up to now and therefore experience and knowledge is sparse in this field. 


\section{Physical and Mathematical Modeling}

\subsection{Governing Equations}

The flow of reacting, multicomponent, compressible fluids is governed by the conservation equations of mass and momentum together with the transport equations for the energy and the different involved species. Let $\rho, t, \mathbf{u}, \sigma, h_{t}, \dot{\mathbf{q}}, Y_{k}, \mathbf{j}_{\mathbf{k}}, \dot{\omega}_{k}$ and $N_{c}$ represent the density, time, velocity vector, stress tensor, total enthalpy, heat flux vector, mass fraction, mass flux vector, source term and the number of species, respectively. The governing equations read:

$$
\begin{gathered}
\frac{\partial \rho}{\partial t}+\nabla \cdot(\rho \mathbf{u})=0, \\
\frac{\partial(\rho \mathbf{u})}{\partial t}+\nabla \cdot(\rho \mathbf{u u})=\nabla \cdot \sigma, \\
\frac{\partial\left(\rho h_{\mathrm{t}}\right)}{\partial t}+\nabla \cdot\left(\rho h_{\mathrm{t}} \mathbf{u}\right)=\frac{\partial p}{\partial t}+\nabla \cdot(\tau \cdot \mathbf{u})-\nabla \cdot \dot{\mathbf{q}}
\end{gathered}
$$

and

$$
\frac{\partial\left(\rho Y_{k}\right)}{\partial t}+\nabla \cdot\left(\rho Y_{k} \mathbf{u}\right)=-\nabla \cdot \mathbf{j}_{\mathbf{k}}+\dot{\omega}_{k} \quad k=1,2, \ldots, N_{c}
$$

The stress tensor $\sigma$ can be expressed for Newtonian fluids as

$$
\sigma=-\left(p+\frac{2}{3} \mu \nabla \cdot \mathbf{u}\right) \mathbf{I}+\mu\left[\nabla \mathbf{u}+(\nabla \mathbf{u})^{\mathrm{T}}\right]=-p \mathbf{I}+\tau
$$

where $\tau$ and $\mu$ are the viscous stress tensor and the dynamic viscosity, respectively. In the energy conservation Eq. (3) the total enthalpy $h_{t}$ represents the sum of the static enthalpy $h$ and the kinetic energy $\frac{1}{2}\|\mathbf{u}\|^{2}$. By applying both Fourier's and Fick's law together with the unitary Lewis-number assumption, i.e., Le $=\kappa\left(\rho c_{p} D\right)^{-1}=1$, for the deduction of the diffusion coefficient $D$, the heat flux vector $\dot{\mathbf{q}}$ and the mass flux vector $\mathbf{j}_{\mathbf{k}}$ can be expressed in the following way:

$$
\begin{gathered}
\dot{\mathbf{q}}=-\frac{\kappa}{c_{p}} \nabla h+\left.\frac{\kappa}{c_{p}} \frac{\partial h}{\partial p}\right|_{T, \mathbf{Y}} \nabla p, \\
\mathbf{j}_{\mathbf{k}}=-\rho D \nabla Y_{k} .
\end{gathered}
$$

Here, $\kappa$ and $c_{p}$ denote the thermal conductivity and the specific heat at constant pressure, respectively. Under subsonic flow conditions - as it is the case here-the pressure contribution in Eq. (6) can be neglected and therefore the divergence of the heat flux $\nabla \cdot \dot{\mathbf{q}}$ can be handled implicitly as the energy equation is written in 
an enthalpy explicit form. In Eqs.(1)-(4), Soret, Dufour and radiation effects are neglected.

\subsection{Numerical Flow Solver}

The finite volume method together with a pressure-based solver formulation is used to discretize the governing equations. In the pressure-based approach, the pressure instead of the density is used as a primary variable. Therefore, the density is recalculated by means of a suitable equation of state $(\mathrm{EoS})$. The pressure is solved with the help of a Poisson-like equation which can be derived from the momentum and continuity equations as [7]:

$$
\frac{\partial \rho}{\partial t}+\nabla \cdot\left[\rho\left(\frac{H_{P}}{a_{P}}\right)\right]-\nabla \cdot\left(\frac{\rho}{a_{P}} \nabla p\right)=0 .
$$

Here, the variables $a_{P}$ and $H_{P}$ result from the discretization of the momentum equation, for further details see, e.g., Ferziger and Peric [7]. As Eq. (8) is elliptical in nature and therefore derived for the incompressible flow regime, slight modifications have to be made to account for the compressibility of the fluid. Following typical pressure correction approaches, the total differential of the density as function of pressure $p$, enthalpy $h$ and species composition $\mathbf{Y}$ can be expressed as:

$$
\mathrm{d} \rho=\left.\frac{\partial \rho}{\partial p}\right|_{h, \mathbf{Y}} \mathrm{d} p+\left.\frac{\partial \rho}{\partial h}\right|_{p, \mathbf{Y}} \mathrm{d} h+\left.\sum_{i=1}^{N_{c}} \frac{\partial \rho}{\partial Y_{i}}\right|_{h, p, Y_{j} \neq Y_{i}} \mathrm{~d} Y_{i}=\psi_{h} \mathrm{~d} p+\left.\frac{\partial \rho}{\partial h}\right|_{p, \mathbf{Y}} \mathrm{d} h+\sum_{i=1}^{N_{c}} \rho_{i} \mathrm{~d} Y_{i} .
$$

Recasting this equation into a Taylor series truncated after the first term, neglecting all variations except the pressure and applying the resulting equation in the time derivative of Eq. (8) yields the following adjusted pressure equation:

$$
\frac{\partial\left(\rho^{n-1}-\psi_{h}^{n-1} p^{n-1}\right)}{\partial t}+\frac{\partial \psi_{h}^{n-1} p}{\partial t}+\nabla \cdot\left[\rho\left(\frac{H_{P}}{a_{P}}\right)\right]-\nabla \cdot\left(\frac{\rho}{a_{P}} \nabla p\right)=0 .
$$

Here, the superscript $n-1$ refers to the last iteration/time step. For solving this pressure equation together with the other governing equations, a segregated solution algorithm is selected. In detail, a so-called PIMPLE approach is applied which is a combination of the Semi-Implicit Method for Pressure Linked Equations (SIMPLE) and the Pressure-Implicit with Splitting of Operators (PISO) method. The solver is implemented in the open-source toolbox OpenFOAM [1]. A more thorough description and discussion can be found in Traxinger et al. [30]. 


\subsection{Thermodynamic Modeling}

For relating and calculating the different thermodynamic properties, appropriate state equations and relations are required. The density and pressure are coupled by means of cubic EoSs which are commonly applied due to their efficiency and acceptable accuracy. The pressure-explicit form of the cubic EoSs reads [21]:

$$
p=\frac{\mathcal{R} T}{v-b}-\frac{a(T)}{v^{2}+u b v+w b^{2}}=\frac{\mathcal{R} T}{v-b}-\frac{a_{c} \alpha(T)}{v^{2}+u b v+w b^{2}} .
$$

Here, $\mathcal{R}$ is the universal gas constant, $T$ is the temperature and $v$ is the molar volume. The parameters $a(T)=a_{c} \alpha(T)$ and $b$ account for the intermolecular attractive and repulsive forces, respectively, and $u$ and $w$ are model constants. Based on Eq. (11), the popular cubic EoSs of Peng and Robinson [19] and Soave, Redlich and Kwong [26] can be deduced as well as the ideal gas equation $(a=b=0)$.

For the consideration of multicomponent mixtures, the concept of a one-fluid mixture in combination with mixing rules is applied [21]

$$
a=\sum_{i=1}^{N_{c}} \sum_{j=1}^{N_{c}} z_{i} z_{j} a_{i j} \quad \text { and } \quad b=\sum_{i=1}^{N_{c}} z_{i} b_{i}
$$

where $z_{i}$ is the mole fraction of the $i$-th component. Pseudo-critical combination rules [22] are employed to determine the off-diagonal elements of $a_{i j}$. The caloric properties are derived consistently by applying the departure function formalism [21]. In this concept, the respective property, e.g., the enthalpy $h$, is divided into an ideal gas (ig) and a real gas (rg) part, i.e.,

$$
h=h^{\mathrm{ig}}(T, \mathbf{z})+\Delta h^{\mathrm{rg}}(T, p, \mathbf{z})=h^{\mathrm{ig}}+R T\left(\int_{0}^{\rho}-\left.T \frac{\partial Z}{\partial T}\right|_{\rho} \frac{\mathrm{d} \rho}{\rho}+Z-1\right)
$$

where $R$ is the specific gas constant. The real-gas contribution can be derived from the applied cubic EoS. The ideal gas part is determined using the seven-coefficient NASA polynomials [9]. For the determination of the viscosity and the thermal conductivity, the empirical correlation of Chung et al. [5] is employed in the real-gas case. In contrast, the approach of Sutherland [27] is used for ideal gases. For considering multicomponent phase separation, a multiphase framework based on the cubic EoSs and the tangent plane concept of Michelsen [13] is applied. This framework relies on the assumption of a local instantaneous thermodynamic equilibrium. For further details, please refer to Traxinger et al. [28, 30]. 


\subsection{Combustion Modeling}

In the applied tabulated combustion models, the mixture fraction $f$ is introduced to describe the combustion progress by means of a passive scalar transport equation

$$
\frac{\partial}{\partial t}(\bar{\rho} \tilde{f})+\frac{\partial}{\partial x_{i}}\left(\bar{\rho} \tilde{u}_{i} \tilde{f}\right)=\frac{\partial}{\partial x_{i}}\left(\left(\frac{\bar{\mu}}{S c}+\frac{\mu_{s g s}}{S c_{t}}\right) \frac{\partial \tilde{f}}{\partial x_{i}}\right)
$$

where $S c$ and $S c_{t}$ are the laminar and turbulent Schmidt number, respectively, and the subscript $s g s$ denotes the contribution of the applied subgrid model due to filtering. Here and in the following, the finite-volume filter is indicated by a bar $\overline{\text { and Favre- }}$ filtering is denoted by a tilde $\tilde{\star}=\bar{\rho} \star / \bar{\rho}$. To model unresolved fluctuations of the mixture fraction, a transport equation for the variance of $f$ is solved [11]

$$
\begin{array}{r}
\frac{\partial}{\partial t}\left(\bar{\rho} \widetilde{f^{\prime \prime 2}}\right)+\frac{\partial}{\partial x_{i}}\left(\bar{\rho} \tilde{u}_{i} \widetilde{f^{\prime \prime 2}}\right)= \\
\frac{\partial}{\partial x_{i}}\left(\left(\frac{\bar{\mu}}{S c}+\frac{\mu_{s g s}}{S c_{t}}\right) \frac{\partial \widetilde{f^{\prime \prime 2}}}{\partial x_{i}}\right)-2 \bar{\rho} \tilde{\chi}+2\left(\frac{\bar{\mu}}{S c}+\frac{\mu_{s g s}}{S c_{t}}\right)\left(\frac{\partial \tilde{f}}{\partial x_{i}}\right)^{2}
\end{array}
$$

where $\chi$ is the scalar dissipation rate modeled according to Domingo et al. [6].

For the generation of the thermo-chemical database, the flamelet concept is applied [20]. Under the assumption of a Damköhler number $D a \gg 1$, the flamelet approach allows to describe the structure of a turbulent nonpremixed flame as a brush of laminar counterflow diffusion flames. By transforming the governing equations into the mixture fraction space and assuming both a constant pressure and a unity Lewis-number, a one-dimensional set of equations can be derived:

$$
\begin{gathered}
\rho \frac{\partial Y_{k}}{\partial t}=\rho \frac{\chi}{2} \frac{\partial^{2} Y_{k}}{\partial f^{2}}+\dot{\omega}_{k}, \\
\rho \frac{\partial h}{\partial t}=\rho \frac{\chi}{2} \frac{\partial^{2} h}{\partial f^{2}} .
\end{gathered}
$$

High-fidelity reaction mechanisms like, for instance, GRI-3.0 [10] are employed to determine the reaction rates. Solving Eqs.(16)-(17) for different scalar dissipation rates up to extinction and a subsequent filtering by means of a presumed probability density function (PDF) yields a suitable library for the LESs of adiabatic combustion. Nonadiabatic effects can be introduced conveniently by assuming a frozen composition or by introducing a semi-permeable wall into the mixture fraction space, see, e.g., Zips et al. [35, 36]. Under real-gas conditions, the pressure dependency of the thermodynamic properties has to be taken into account in the tabulation [34].

In contrast to presumed PDF approaches, transported PDF methods allow for the evaluation of the PDF $\mathcal{P}_{\text {sgs }}$ by means of a transport equation [8] 


$$
\begin{array}{r}
\frac{\partial \bar{\rho} \widetilde{\mathcal{P}}_{s g s}}{\partial t}+\frac{\partial \bar{\rho} \tilde{u}_{i} \widetilde{\mathcal{P}}_{s g s}}{\partial x_{i}}+\sum_{\alpha=1}^{N_{c}} \frac{\partial}{\partial \Psi_{\alpha}}\left(\bar{\rho} \dot{\omega}_{\alpha} \widetilde{\mathcal{P}}_{s g s}\right)= \\
\frac{\partial}{\partial x_{i}}\left[\left(\frac{\mu}{S c}+\frac{\mu_{s g s}}{S c_{t}}\right) \frac{\partial \widetilde{\mathcal{P}}_{s g s}}{\partial x_{i}}\right]-\frac{\bar{\rho}}{\tau_{s g s}} \sum_{\alpha=1}^{N_{c}} \frac{\partial}{\partial \Psi_{\alpha}}\left[\left(\Psi_{\alpha}-\widetilde{\phi}_{\alpha}\right) \widetilde{\mathcal{P}}_{s g s}\right]
\end{array}
$$

where $\Psi$ denotes the thermo-chemical state space. This transport equation is usually solved using statistic methods. In the present work, the Eulerian stochastic fields (ESF) method proposed by Valiño [33] is employed. A more thorough description and discussion can be found in Zips et al. [35].

\section{Results and Discussion}

\subsection{Thermodynamics}

Under supercritical pressure conditions, the dense-gas approach is widely-used in the LRE community which implies the assumption of a sole single-phase state. For the pioneering works with only a single component like, e.g., Zong et al. [37], this assumption is perfectly valid. However, in multicomponent mixtures, as it is the case during injection and combustion under LRE conditions, the multiphase region is determined by a critical locus rather than a distinct critical point. Due to the nonlinear behavior of real-gas mixtures this locus can exceed the critical values of the pure components by orders of magnitude, especially with respect to the pressure, see Fig. 1 left.

A-posteriori investigations of the mixture states of a binary hydrogen/nitrogen test case [18] at cryogenic temperatures showed first evidence of phase separation under initially supercritical conditions [14]. In Fig. 1 right, simulation data of this test case are scattered into a temperature composition diagram and superimposed onto the mixture two-phase region. In the composition range of $0.15 \leq z_{\mathrm{H}_{2}} \leq 0.35$ the mixture states penetrate the vapor-liquid equilibrium (VLE) rendering the single-phase assumption invalid. As there is no profound evidence for this statement, additional test cases were defined in close cooperation with the ITLR at the University of Stuttgart

Fig. 1 Critical locus for different alkane mixtures (left) and a-posteriori evaluation of the LES results of a hydrogen/nitrogen test case [14] with respect to the VLE of the binary mixture (right)
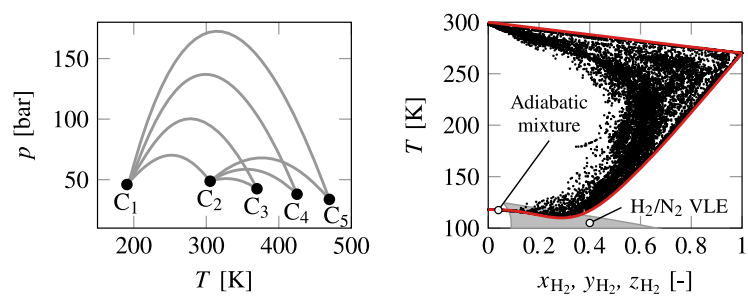
where an appropriate experimental test facility is available [2]. In this campaign, initially supercritical $n$-hexane $\left(\mathrm{C}_{6} \mathrm{H}_{14}\right)$ was injected into a pressurized nitrogen atmosphere at three different total temperatures $\left(T_{t, \mathrm{C}_{6} \mathrm{H}_{14}}=[480,560,600] \mathrm{K}\right)$. In the experiments, simultaneous shadowgraphy and elastic light scattering (ELS) was conducted to visualize both the jet structure and the phase separation process. LESs employing the multicomponent VLE model as thermodynamic closure have been used for the numerical investigation. Both the experiments and the simulations show similar phase separation phenomena at the respective temperature and a transition from a dense-gas mixture $\left(T_{t, \mathrm{C}_{6} \mathrm{H}_{14}}=600 \mathrm{~K}\right)$ to a spray-like jet $\left(T_{t, \mathrm{C}_{6} \mathrm{H}_{14}}=480 \mathrm{~K}\right)$ proving the presence of phase separation under high-pressure conditions depending on the injection temperature, see Fig. 2 and for further details Traxinger et al. [29]. Applying the same VLE model, the single-phase stability in a high-pressure methane combustion case was investigated. The analysis showed strong phase separation phenomena on the oxidizer-rich side and a large spatial extent inside the flame, see Fig. 3. The phase separation process is triggered due to the low temperatures and the presence of water [32] originating from the combustion and subsequent diffusion processes.
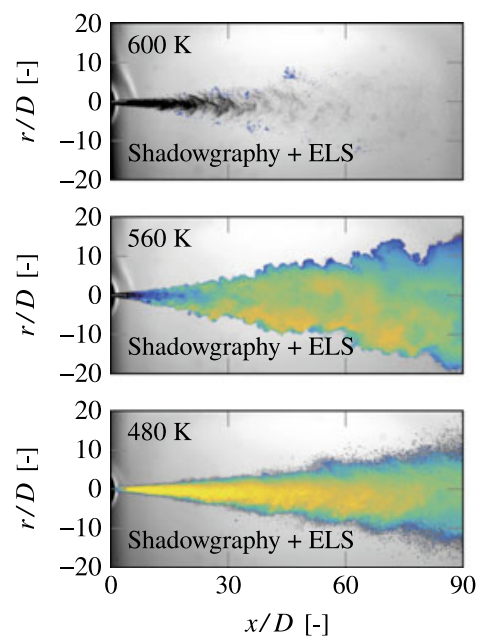
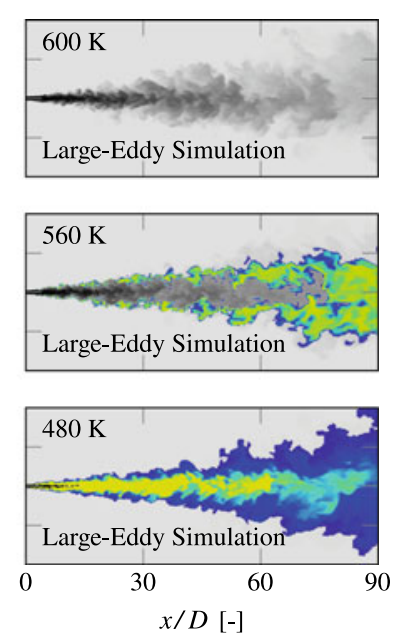

Fig. 2 Comparison of experimental (left) and numerical (right) snapshots of the n-hexane jet at three different temperatures $\left(T_{t, \mathrm{C}_{6} \mathrm{H}_{14}}=[600,560,480] \mathrm{K}\right)$. The LES results are shown by means of the instantaneous vapor fraction superimposed onto the temperature field. Reprinted figure with permission from Traxinger et al. [29]. Copyright (2020) by the American Physical Society 

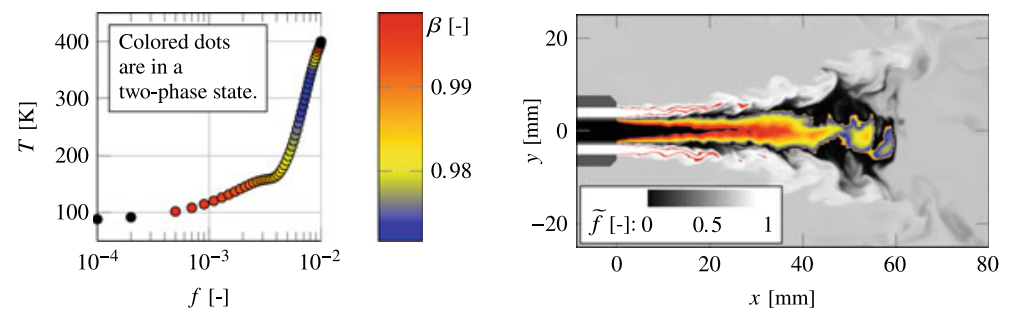

Fig. 3 Mixture-induced phase separation in a high-pressure methane flame. Left: Flamelet solution on the oxidizer-rich side. Right: Instantaneous LES result

\subsection{Combustion}

The increased interest in reusable, methane-fired engines sets new demands into the development and improvement of CFD tools. Although a lot of research effort has been invested in the field of nonadiabatic rocket combustion chamber modeling in recent years, most of the past studies investigate hydrogen combustion. Furthermore, the dimensions of the test chambers, e.g., the Pennstate pre-burner, were not really rocket-engine typical and therefore the results cannot be fully used as a blueprint for more application-relevant studies. Therefore, two new oxygen/methane combustion chamber test cases have been defined by the group of Prof. Haidn at the Technical University of Munich featuring more application-related chamber characteristics, e.g., element-element and element-wall distances: a single-element combustion chamber [4] and a 7-element combustor [25]. Using the single-element combustion chamber as a reference case, the influence of the turbulence wall model on the predicted wall heat flux has been investigated. In Fig. 4 left, a general impression of the temperature field is given at different axial positions. The quadratic chamber cross section clearly influences the axial development of the flame. The comparison of the predicted heat flux with the experiment, see Fig. 4 right, shows that all
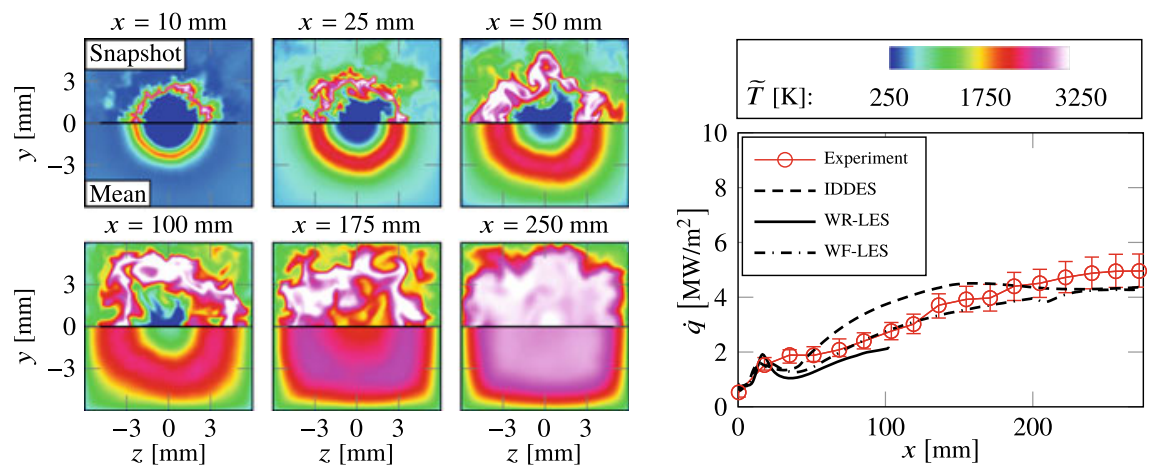

Fig. 4 Large-Eddy Simulation results of the single-element combustion test case 

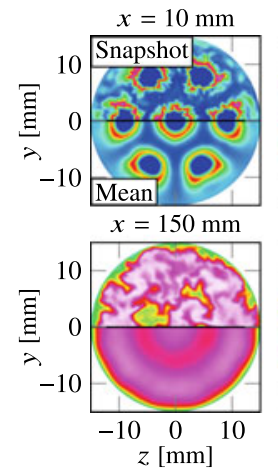

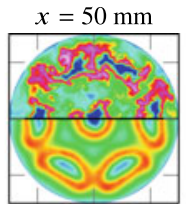

$x=200 \mathrm{~mm}$

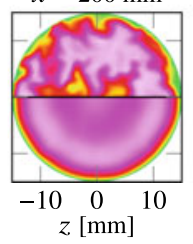

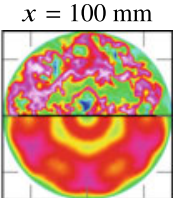

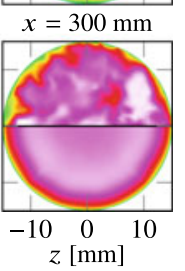

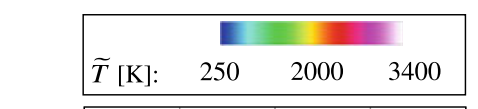

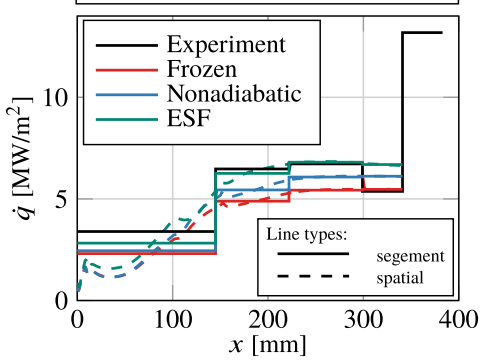

Fig. 5 Large-Eddy Simulation results of the multi-element combustion test case

models capture the basic trend. Overall, the wall-modeled LES (WF-LES) shows the best results and was therefore employed for further studies on the influence of the combustion model. The 7-element test case was used to compare three different combustion models namely two presumed PDF approaches and one transported PDF approach. In Fig. 5 left, temperature contours at different axial positions are shown indicating the gradual flame-flame interaction with increasing axial direction. In terms of the predicted wall heat flux, see Fig. 5 right, the ESF method shows the best results followed by the nonadiabatic and the frozen flamelet model. In Fig. 6 scatter plots of temperature and selected mass fractions are shown for the different combustion models revealing clear differences. As expected, the frozen model reproduces the composition of the nominal flamelet. The nonadiabatic solution shows stronger scattering in terms of species mass fraction but is different to the ESF solution.
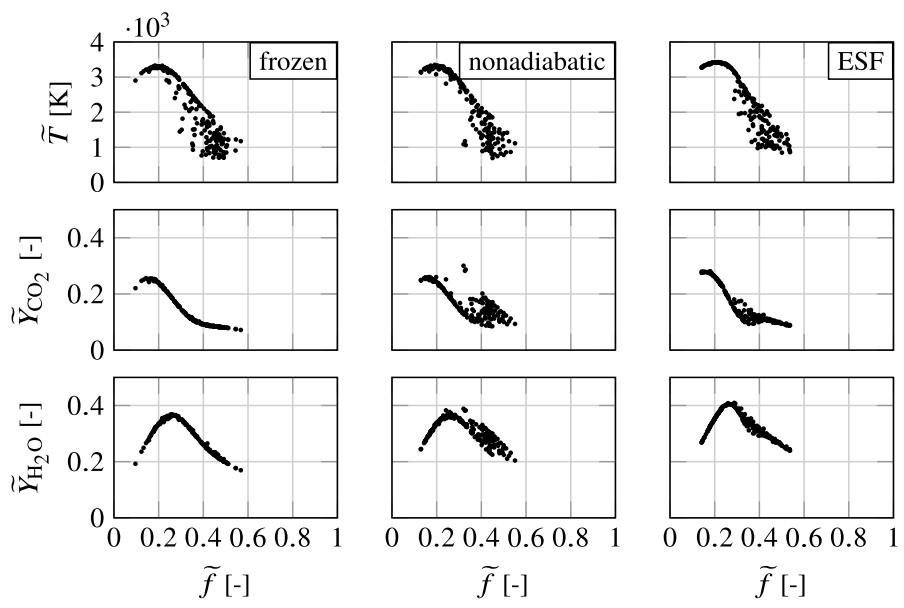

Fig. 6 Scatter plot of the multi-element test case at $x=200 \mathrm{~mm}$ 


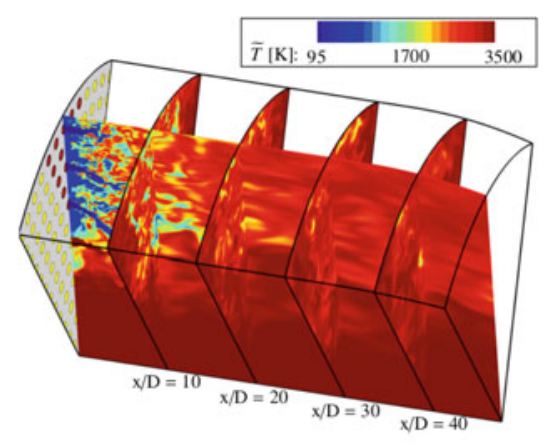

a) Instantaneous temperature field.

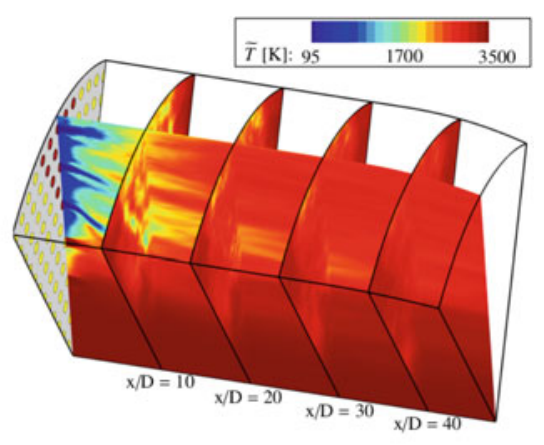

b) Mean temperature field.

Fig. 7 Large-Eddy Simulation result of the temperature field of the full-scale TCD

Finally, a 60 degrees section of a full-scale methane-fired thrust chamber demonstrator (TCD) defined by ArianeGroup has been investigated focusing on flameflame interaction, see Fig. 7. The operating conditions of the TCD are inspired by the Prometheus engine. The LES result reveals strong flame-flame interaction starting at $x / D \approx 3$. As a consequence, temperatures below $1500 \mathrm{~K}$ are only present up to $x / D \approx 10$ which is very different to the investigation of a single injection element under identical operating conditions where flame-flame interaction is inherently missing [31].

\section{Conclusion}

For the development of future rocket engines, CFD is an indispensable tool. Together with suitable experiments, numerical investigations can provide insight into the flow field and increase the knowledge and understanding of the complex on-going processes. In the present work, the focus was put on the validation of thermodynamics and combustion modeling. By means of LESs it could be shown that single-phase instabilities can occur during mixing and combustion under LRE-like conditions rendering the dense-gas approach invalid. For a single- and a multi-element modelcombustor, numerical heat flux predictions were compared to experimental data. Wall-modeled LES together with a high-fidelity combustion model is a promising candidate for an accurate prediction.

Acknowledgements Financial support was provided by the German Research Foundation (Deutsche Forschungsgemeinschaft-DFG) in the framework of the Sonderforschungsbereich Transregio 40 and Munich Aerospace (www.munich-aerospace.de). The authors gratefully acknowledge the Gauss Centre for Supercomputing e.V. (GCS) for funding this project by providing computing time on the GCS Supercomputer SuperMUC at the Leibniz Supercomputing Centre. 


\section{References}

1. Openfoam 4.1. https://openfoam.org/

2. Baab, S., Förster, F.J., Lamanna, G., Weigand, B.: Combined elastic light scattering and twoscale shadowgraphy of near critical fuel jets. In: 26th Annual Conference on Liquid Atomization and Spray Systems (2014)

3. Bellan, J.: Theory, modeling and analysis of turbulent supercritical mixing. Combust. Sci. Technol. 178(1-3), 253-281 (2006)

4. Celano, M., Silvestri, S., Schlieben, G., Kirchberger, C., Haidn, O., Knab, O.: Injector characterization for a gaseous oxygen-methane single element combustion chamber. In: Progress in Propulsion Physics (2016)

5. Chung, T., Ajlan, M., Lee, L., Starling, K.E.: Generalized multiparameter correlation for nonpolar and polar fluid transport properties. Ind. Eng. Chem. Res. 27(4), 671-679 (1988)

6. Domingo, P., Vervisch, L., Veynante, D.: Large-eddy simulation of a lifted methane jet flame in a vitiated coflow. Combust. Flame 152(3), 415-432 (2008)

7. Ferziger, J.H., Peric, M.: Computational Methods for Fluid Dynamics. Springer, Berlin (2002)

8. Gerlinger, P.: Numerische Verbrennungssimulation. Springer, Berlin (2005)

9. Goos, E., Burcat, A., Ruscic, B.: Report ANL 05/20 TAE 960. Technical Report (2005). http:// burcat.technion.ac.il/dir

10. GRI-Mech 3.0.: University of California at Berkeley. http://www.me.berkeley.edu/gri-mech (2000)

11. Kemenov, K.A., Wang, H., Pope, S.B.: Modelling effects of subgrid-scale mixture fraction variance in les of a piloted diffusion flame. Combust. Theory Modell. 16(4), 611-638 (2012)

12. Lacaze, G., Oefelein, J.C.: A non-premixed combustion model based on flame structure analysis at supercritical pressures. Combust. Flame 159(6), 2087-2103 (2012)

13. Michelsen, M.L.: The isothermal flash problem. part i. stability. Fluid Phase Equilib. 9(1), 1-19 (1982)

14. Müller, H., Pfitzner, M., Matheis, J., Hickel, S.: Large-eddy simulation of coaxial $\ln 2 / g h 2$ injection at trans-and supercritical conditions. J. Propuls. Power 32(1), 46-56 (2016)

15. Muthukumaran, C., Vaidyanathan, A.: Experimental study of elliptical jet from sub to supercritical conditions. Phys. Fluids 26(4), 044104 (2014)

16. Oefelein, J.C.: Advances in modeling supercritical fluid behavior and combustion in highpressure propulsion systems. In: AIAA Scitech 2019 Forum (2019)

17. Oefelein, J.C., Yang, V.: Modeling high-pressure mixing and combustion processes in liquid rocket engines. J. Propuls. Power 14(5), 843-857 (1998)

18. Oschwald, M., Schik, A., Klar, M., Mayer, W.: Investigation of coaxial $\ln 2 /$ gh2-injection at supercritical pressure by spontaneous raman scattering. In: 35th Joint Propulsion Conference and Exhibit (1999)

19. Peng, D.Y., Robinson, D.B.: A new two-constant equation of state. Ind. Eng. Chem. Fundam. 15(1), 59-64 (1976)

20. Peters, N.: Turbulent combustion. Cambridge University Press, Cambridge (2005)

21. Poling, B.E., Prausnitz, J.M., O'Connell, J.P.: the properties of gases and liquids. McGraw-Hill, New York (2001)

22. Reid, R.C., Prausnitz, J.M., Poling, B.E.: the properties of liquids and gases. McGraw-Hill, New York (1987)

23. Ribert, G., Zong, N., Yang, V., Pons, L., Darabiha, N., Candel, S.: Counterflow diffusion flames of general fluids: oxygen/hydrogen mixtures. Combust. Flame 154(3), 319-330 (2008)

24. Roy, A., Joly, C., Segal, C.: Disintegrating supercritical jets in a subcritical environment. J. Fluid Mech. 717, 193-202 (2013)

25. Silvestri, S., Celano, M.P., Schlieben, G., Haidn, O.J.: Characterization of a multi-injector gox/ch4 combustion chamber. In: 52nd AIAA/SAE/ASEE Joint Propulsion Conference, p. $4992(2016)$

26. Soave, G.: Equilibrium constants from a modified redlich-kwong equation of state. Chem. Eng. Sci. 27(6), 1197-1203 (1972) 
27. Sutherland, W.: Lii. the viscosity of gases and molecular force. Lond. Edinb. Dubl. Phil. Mag. J. Sci. 36(223), 507-531 (1893)

28. Traxinger, C., Banholzer, M., Pfitzner, M.: Real-gas effects and phase separation in underexpanded jets at engine-relevant conditions. In: 2018 AIAA Aerospace Sciences Meeting (2018)

29. Traxinger, C., Pfitzner, M., Baab, S., Lamanna, G., Weigand, B.: Experimental and numerical investigation of phase separation due to multi-component mixing at high-pressure conditions. Phys. Rev. Fluids 4(7), 074303 (2019)

30. Traxinger, C., Zips, J., Banholzer, M., Pfitzner, M.: A pressure-based solution framework for sub-and supersonic flows considering real-gas effects and phase separation under enginerelevant conditions. Comput. Fluids 202, 104452 (2020)

31. Traxinger, C., Zips, J., Pfitzner, M.: Large-eddy simulation of a multi-element lox $/ \mathrm{ch}_{4}$ thrust chamber demonstrator of a liquid rocket engine. In: 8th European Conference for Aeronautics and Aerospace Sciences (2019)

32. Traxinger, C., Zips, J., Pfitzner, M.: Single-phase instability in non-premixed flames under liquid rocket engine relevant conditions. J. Propuls. Power 35(4), 675-689 (2019)

33. Valiño, L.: Field monte carlo formulation for calculating the probability density function of a single scalar in a turbulent flow. Flow Turbul. Combust. 60(2), 157-172 (1998)

34. Zips, J., Müller, H., Pfitzner, M.: Efficient thermo-chemistry tabulation for non-premixed combustion at high-pressure conditions. Flow Turbul. Combust. 101(3), 821-850 (2018)

35. Zips, J., Traxinger, C., Breda, P., Pfitzner, M.: Les of a 7-element gox/gch4 subscale combustion chamber using presumed and transported pdf methods. J. Propuls. Power 35(4), 747-764 (2019)

36. Zips, J., Traxinger, C., Pfitzner, M.: Time-resolved flow field and thermal loads in a singleelement gox/gch4 rocket combustor. Int. J. Heat Mass Transf. 143, 118474 (2019)

37. Zong, N., Meng, H., Hsieh, S.Y., Yang, V.: A numerical study of cryogenic fluid injection and mixing under supercritical conditions. Phys. Fluids 16(12), 4248-4261 (2004)

Open Access This chapter is licensed under the terms of the Creative Commons Attribution 4.0 International License (http://creativecommons.org/licenses/by/4.0/), which permits use, sharing, adaptation, distribution and reproduction in any medium or format, as long as you give appropriate credit to the original author(s) and the source, provide a link to the Creative Commons license and indicate if changes were made.

The images or other third party material in this chapter are included in the chapter's Creative Commons license, unless indicated otherwise in a credit line to the material. If material is not included in the chapter's Creative Commons license and your intended use is not permitted by statutory regulation or exceeds the permitted use, you will need to obtain permission directly from the copyright holder.

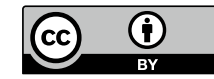

